\title{
Efficacy of Tibolone and Isoflavones in the Management of Postmenopausal Symptoms
}

\author{
Suresh Chandra Mandal ${ }^{1}$, Soumyakanti Barman ${ }^{2}$, Sandip Lahiri $^{3}$, Sudip Ranjan Pal ${ }^{4}$ \\ Department of Obstetrics \& Gynaecology, Malda Medical College, Malda, West Bengal, India
}

\begin{abstract}
:
Background: After the recent publication of results from two large prospective studies, Women's Health Initiativeand Heart and Estrogen/ Progestin Replacement Therapy (HERS) indicating increased risk with hormone replacement therapy (HRT) for menopausal symptoms, HRT is no longer recommended for asymptomatic postmenopausal women. Tibolone has emerged as an attractive alternative for treatment of perimenopausal women. Interest in the use of Isoflavones, the most common from of phytoestrogens for treatment of menopausal symptoms has been encouraged by observation of lower prevalence of menopausal symptoms. The present study attempts to evaluate the efficacy and side effects of tibolone and isoflavone in the treatment of menopausal symptoms.

Methods: This prospective comparative study assessed the usefulness of tibolone and isoflavone for the management of menopausal symptoms among 80 postmenopausal symptoms. The patients were asked about symptomatic improvements ; detailed physical examination were carried out ; TVS and lipid profile were done initially and at 1,6 and 12 months.

Result : Improvement in vasomotor, genitourinary symptoms and joint pain were significantly higher in tibolone group in comparison to isoflavone in most of the follow up visits and after the completion of treatment.Blood pressure records did not show any change with the use of both the drugs over 12 months. Incidence of breakthrough bleeding was nil in isoflavone and nominal in tibolone group . No significant adverse effect on lipid profile was found in both the groups .
\end{abstract}

Conclusion: Efficacy of tibolone was found to be significantly better than the isoflavones in management of postmenopausal symptoms.

Keywords : Tibolone, Isoflavone, Postmenopausal symptoms

\section{INTRODUCTION}

The world population is not only growing but also graying. So maintenance of peri and postmenopausal health is of paramount importance to minimize the economic impact on our society. Due to fear and dislike of adverse effects, as well as possible long term risk of HRT, there is increasing interest in effective \& safe alternatives to HRT for menopausal problems. Tibolone, a synthetic steroid, represents an advance in hormone replacement therapy, offering an attractive option for treatment of menopausal women. On the other hand, plant derived substances structurally related to estrogens that have been shown to bind to estrogen receptors, commonly termed phytoestrogens are currently used by many women as alternatives to HRT . Of this isoflavones, the most common from of phytoestrogens, primarily found in soy beans and their by products are most widely used and studied class. The specific objectives of the study are to evaluate the efficacy of tibolone and isoflavones in the management of postmenopausal symptoms, in relation to alleviation of symptoms and side effects

\section{MATERIALS AND MethodS}

A total of 80 postmenopausal women seeking help for relief of menopausal symptoms were recruited for this prospective comparative study between tibolone $(2.5 \mathrm{mg})$ and isoflavones $(100 \mathrm{mg})$ in the department of Obstetrics and Gynecology, National medical college and hospital, Kolkata, between June 2009 to September 2010. Ten women from tibolone and 7 women from isoflavone group were lost during follow up and foregoing description and analysis pertains to 30 and 33 women from the respective groups. Patient were advised to come for follow up at 1 month, 6 month and 12 months after initiation of therapy. Patient were enquired about symptomatic improvement

${ }^{1}$ Corresponding Author: sureshmondal77@gmail.com 


\section{American Research Journal of Medicine And Surgery, Volume 1, Issue 2, 2015}

ISSN 2379-8955

(vasomotor symptoms, urinary symptoms, vaginal symptoms and joint pain ); a detailed physical examination was performed in each visit. Fasting lipid profile and transvaginal sonography were performed initially and after six month and twelve month. Statisrical analysis was performed using SPSS for windows, Student $t$ test and chi squere test were used wherever appropriate. A p value of $<0.05$ was taken as statistically significant.

\section{RESULT AND ANALYSIS}

Table1. Effect of Treatment on Vasomotor Symptoms

\begin{tabular}{|c|c|c|c|c|c|c|c|c|c|c|c|}
\hline & Response & \begin{tabular}{|l|} 
after 1 \\
month \\
\end{tabular} & & Response & \begin{tabular}{|c|} 
after 6 \\
months \\
\end{tabular} & & Response & $\begin{array}{l}\text { after } 12 \\
\text { months }\end{array}$ & & Response & \\
\hline & Complete & Partial & Nil & Complete & Partial & Nil & Complete & Partial & Nil & $\begin{array}{c}\text { Overall } \\
\text { improveme } \\
\text { nt }\end{array}$ & Nil \\
\hline $\begin{array}{c}\begin{array}{c}\text { Tibolone } \\
(\mathrm{N}=26)\end{array} \\
\end{array}$ & $\begin{array}{c}10(38.46 \\
\%)\end{array}$ & \begin{tabular}{|c|}
$\begin{array}{c}13(50 \\
\%)\end{array}$ \\
\end{tabular} & $\begin{array}{c}3(11.54 \\
\%)\end{array}$ & $\begin{array}{c}16(61.54 \\
\%)\end{array}$ & $\begin{array}{c}9(34.64 \\
\%)\end{array}$ & $\begin{array}{c}1(3.85 \\
\%)\end{array}$ & $\begin{array}{c}17(65.38 \\
\%)\end{array}$ & $8(30 \%)$ & $\begin{array}{c}1(3.85 \\
\%)\end{array}$ & $25(95 \%)$ & $1(3.85 \%)$ \\
\hline \begin{tabular}{|c|} 
Isoflavon \\
es \\
$(\mathrm{N}=25)$
\end{tabular} & 0 & $\begin{array}{c}10(40 \\
\%)\end{array}$ & $15(60 \%)$ & $0(0 \%)$ & $15(50 \%)$ & $\begin{array}{c}10(40 \% \\
)\end{array}$ & $2(8 \%)$ & $15(60 \%)$ & $8(28 \%)$ & $17(68 \%)$ & $8(28 \%)$ \\
\hline $\begin{array}{c}\text { Statistical } \\
\text { analysis }\end{array}$ & & & & & & & $\begin{array}{c}\mathrm{X}^{2}(\text { Yates } \\
\text { corrected }) \\
=15.55 \\
\mathrm{p}=.00007 \\
89\end{array}$ & $\begin{array}{c}\text { Significa } \\
\text { nt }\end{array}$ & & $\begin{array}{c}\mathrm{X}^{2}(\text { Yates } \\
\text { corrected }) \\
=5.15, \\
\mathrm{p}=0.0232 \\
\text { degree of } \\
\text { freedom }=1\end{array}$ & $\begin{array}{c}\text { Significa } \\
\text { nt }\end{array}$ \\
\hline
\end{tabular}

Table2. Effect of Treatment on Genital Symptoms

\begin{tabular}{|c|c|c|c|c|c|c|c|c|c|c|c|}
\hline & \begin{tabular}{|l|} 
Respon \\
se
\end{tabular} & $\begin{array}{ll}\text { after } 1 \\
\text { month }\end{array}$ & & $\begin{array}{l}\text { Respons } \\
\text { e }\end{array}$ & $\begin{array}{ll}\text { after } 6 \\
\text { month }\end{array}$ & & $\begin{array}{l}\text { Respo } \\
\text { nse }\end{array}$ & $\begin{array}{l}\text { after } 12 \\
\text { month }\end{array}$ & & Response & \\
\hline & $\begin{array}{l}\text { Comple } \\
\text { te }\end{array}$ & Partial & Nil & Complet & Partial & Nil & $\begin{array}{l}\text { Compl } \\
\text { ete }\end{array}$ & Partial & Nil & $\begin{array}{l}\text { Overall } \\
\text { improvement }\end{array}$ & Nil \\
\hline \begin{tabular}{|l|} 
Tibolone \\
$(\mathrm{N}=26)$
\end{tabular} & \begin{tabular}{|l} 
\\
$(7.14 \%)$ \\
\end{tabular} & $7(50 \%)$ & $\begin{array}{l}6(42.86 \% \\
)\end{array}$ & \begin{tabular}{|l|}
$6(42.86$ \\
$\%)$ \\
\end{tabular} & $\begin{array}{l}6(42.86 \\
\%)\end{array}$ & $\begin{array}{l}2(14.2 \\
9 \%) \\
\end{array}$ & $\begin{array}{l}8(57.1 \\
4) \\
\end{array}$ & $4(28.57)$ & $2(14.29)$ & $12(85.71 \%)$ & $2(14.29 \%)$ \\
\hline \begin{tabular}{|l|} 
Isoflavo \\
nes \\
$(\mathrm{N}=25)$
\end{tabular} & 0 & $\begin{array}{l}2(14.29 \\
\%)\end{array}$ & $\begin{array}{l}12(85.71 \\
\%)\end{array}$ & 0 & $\begin{array}{l}3(21.43 \\
\%)\end{array}$ & $\begin{array}{l}11(78 . \\
67 \%)\end{array}$ & 0 & $\begin{array}{l}3(21.43 \\
\%)\end{array}$ & $\begin{array}{l}11(78.6 \\
7 \%)\end{array}$ & $3(21.43 \%)$ & $11(78 \%)$ \\
\hline$\left|\begin{array}{l}\text { Statistica } \\
1 \text { analysis }\end{array}\right|$ & & & & & $\begin{array}{l}\text { Significa } \\
\text { nt }\end{array}$ & & $\begin{array}{l}\text { Signifi } \\
\text { cant }\end{array}$ & & & $\begin{array}{c}\mathrm{X}^{2}=9.19 \\
\mathrm{p}=.00243 \\
\quad \text { Degree of } \\
\text { freedom }=1\end{array}$ & Significant \\
\hline
\end{tabular}

Table3. Effect of Treatment on Urinary Symptoms

\begin{tabular}{|c|c|c|c|c|c|c|c|c|c|c|c|}
\hline & Response & \begin{tabular}{|l} 
after \\
month
\end{tabular} & 1 & $\begin{array}{l}\text { Respons } \\
\text { e }\end{array}$ & $\begin{array}{|ll|}\text { after } & 6 \\
\text { month } & \\
\end{array}$ & & Response & \begin{tabular}{|ll} 
after & 12 \\
month &
\end{tabular} & & Response & \\
\hline & $\begin{array}{l}\text { Complet } \\
\text { e }\end{array}$ & Partial & Nil & $\begin{array}{l}\text { Complet } \\
\text { e }\end{array}$ & Partial & Nil & $\begin{array}{l}\text { Complet } \\
\text { e }\end{array}$ & Partial & Nil & \begin{tabular}{|l|} 
Overall \\
improvement
\end{tabular} & Nil \\
\hline $\begin{array}{l}\text { Tibolone } \\
(\mathrm{N}=26)\end{array}$ & $1(12.5 \%)$ & $3(37.5 \%)$ & $4(50 \%)$ & $2(25 \%)$ & $4(50 \%)$ & $2(25 \%)$ & $3(37.5 \%)$ & $3(37.5 \%)$ & $\begin{array}{l}2(25 \\
\%)\end{array}$ & $6(75 \%)$ & $2(25 \%)$ \\
\hline \begin{tabular}{|l|} 
Isoflavone \\
$\mathrm{s}$ \\
$(\mathrm{N}=25)$ \\
\end{tabular} & 0 & $2(16.66 \%)$ & $10(83.33 \%$ & 0 & $3(25 \%)$ & $9(75 \%)$ & 0 & $3(25 \%)$ & $\mid \begin{array}{l}9(75 \\
\%)\end{array}$ & $3(25 \%)$ & $9(75 \%)$ \\
\hline $\begin{array}{l}\text { Statistical } \\
\text { analysis }\end{array}$ & & Significant & & & $\mid \begin{array}{l}\text { Significan } \\
\mathrm{t}\end{array}$ & & & $\begin{array}{l}\text { Significan } \\
t\end{array}$ & & $\begin{array}{l}\mathrm{x}^{2}(\text { Yates } \\
\text { corrected }) \\
3.04, \quad \mathrm{p}=.081 \\
\text { Degree } \\
\text { freedom }=1\end{array}$ & $\begin{array}{l}\text { Significa } \\
\text { nt }\end{array}$ \\
\hline
\end{tabular}


American Research Journal of Medicine and Surgery, Volume 1, Issue 2, 2015

ISSN 2379-8955

Table4. Effect of Treatment on Joint Pain

\begin{tabular}{|c|c|c|c|c|c|c|c|c|c|c|c|}
\hline & $\begin{array}{l}\text { Respons } \\
\text { e }\end{array}$ & $\begin{array}{ll}\text { after } & 1 \\
\text { month }\end{array}$ & & $\begin{array}{l}\text { Respons } \\
\mathrm{e}\end{array}$ & $\begin{array}{ll}\text { after } & 6 \\
\text { month }\end{array}$ & & Response & $\begin{array}{l}\text { after 12 } \\
\text { month }\end{array}$ & & Response & \\
\hline & $\begin{array}{l}\text { Complet } \\
\text { e }\end{array}$ & Partial & Nil & Complet & Partial & Nil & Complete & Partial & Nil & $\begin{array}{l}\text { Overall } \\
\text { improvemen } \\
t\end{array}$ & Nil \\
\hline $\begin{array}{l}\text { Tibolone } \\
(\mathrm{N}=26)\end{array}$ & 0 & $2(25 \%)$ & $6(75 \%)$ & $2(25 \%)$ & $3(37.5 \%)$ & $\begin{array}{l}3(37.5 \% \\
)\end{array}$ & $4(50 \%)$ & $\begin{array}{l}3(37.5 \\
\%)\end{array}$ & $1(12.5 \%$ & $7(87.5 \%)$ & $1(12.5 \%)$ \\
\hline $\begin{array}{l}\text { Isoflavone } \\
\mathrm{s} \\
(\mathrm{N}=25)\end{array}$ & 0 & 0 & $8(100 \%$ & 0 & $2(25 \%)$ & $6(75 \%)$ & 0 & $2(25 \%)$ & $6(75 \%)$ & $2(25 \%)$ & $6(75 \%)$ \\
\hline $\begin{array}{l}\text { Statistical } \\
\text { analysis }\end{array}$ & & & & & & & & & & $\begin{array}{l}\mathrm{X}^{2}(\text { Yates } \\
\text { corrected }) \\
=4.06, \\
\mathrm{p}=.0438 \\
\text { degree of } \\
\text { freedom }=1\end{array}$ & $\begin{array}{l}\text { Significan } \\
t\end{array}$ \\
\hline
\end{tabular}

Table5. Side Effects of Tibolone and Isoflavone

\begin{tabular}{|c|c|c|}
\hline Complaints & Tibolone $(\mathrm{N}=30)$ & Isoflavone $(\mathrm{N}=33)$ \\
\hline Gastrointestinal upset & $4(13.3 \%)$ & $6(18.18 \%)$ \\
\hline Weight gain & $2(6.66 \%)$ & Nil \\
\hline Breast complaints & Nil & Nil \\
\hline Vaginal bleeding( after 12 month) & $2(14.3 \%)$ & Nil \\
\hline
\end{tabular}

Table6. effect on BP after 12 month of treatment

\begin{tabular}{|l|l|l|l|}
\hline & Response & Tibolone & Isoflavone \\
\hline \multirow{4}{*}{ Systolic BP } & Pre treatment & $123.26 \pm 12.11$ & $124.72 \pm 11.7$ \\
\cline { 2 - 4 } & Post treatment & $124.2 \pm 11.13$ & $125.45 \pm 10.35$ \\
\cline { 2 - 4 } & Statistical difference & $\begin{array}{l}\mathrm{t}=0.31[\mathrm{DF}=58], \mathrm{p}=0.75 \\
\text { Non significant }\end{array}$ & $\begin{array}{l}\mathrm{t}=0.78[\mathrm{DF}=64], \mathrm{t}=0.27 \\
\text { Non significant }\end{array}$ \\
\hline \multirow{5}{*}{ Diastolic BP } & Pre treatment & $78.46 \pm 10.09$ & $80.06 \pm 9.23$ \\
\cline { 2 - 4 } & Post treatment & $79.6 \pm 10.1$ & $80.55 \pm 7.60$ \\
\cline { 2 - 4 } & statistical difference & $\begin{array}{l}\mathrm{t}=1.39[\mathrm{DF}=88], \mathrm{p}=0.17 \\
\text { Non significant }\end{array}$ & $\begin{array}{l}\mathrm{t}=0.24[\mathrm{DF}=64], \mathrm{p}=0.82 \\
\text { Non significant }\end{array}$ \\
\hline
\end{tabular}

Table7. Incidence of Breakthrough Bleeding

\begin{tabular}{|l|l|l|}
\hline Duration of treatment & Tibolone $(\mathrm{N}=14)$ & Isoflavone $(\mathrm{N}=16)$ \\
\hline 1 month & $7(50 \%)$ & $2(12.5 \%)$ \\
\hline 6 month & $3(21 \%)$ & 0 \\
\hline 12 month & $2(14.3 \%)$ & 0 \\
\hline
\end{tabular}

Table8. Change in Endometrial Thickness (Mm)

\begin{tabular}{|c|c|c|}
\hline Duration of treatment & Tibolone (N=14) & Isoflavone $(\mathrm{N}=16)$ \\
\hline At recruitment & $3.25 \pm .89$ & $3.16 \pm .73$ \\
\hline After one year & $3.4 \pm .49$ & $3.11 \pm .53$ \\
\hline Statistical difference & $\begin{array}{l}\mathrm{t}=0.55[\mathrm{DF}=26], \mathrm{p}=0.55 \\
\text { Non significant }\end{array}$ & $\begin{array}{l}\mathrm{t}=0.22[\mathrm{DF}=30], \mathrm{p}=0.83 \\
\text { Non significant }\end{array}$ \\
\hline
\end{tabular}


American Research Journal of Medicine And Surgery, Volume 1, Issue 2, 2015

ISSN 2379-8955

Table9. Alteration in Lipid Parameters

\begin{tabular}{|c|c|c|c|c|}
\hline \multirow{2}{*}{ Lipid parameters } & \multicolumn{2}{|l|}{ Tibolone } & \multicolumn{2}{|l|}{ Isoflavones } \\
\hline & Baseline & After 12 month & Baseline & After 12 month \\
\hline \multirow[b]{2}{*}{ Total cholesterol } & $169.63 \pm 26.12$ & $149 \pm 23.01$ & $147.12 \pm 21.53$ & $146.12 \pm 18.84$ \\
\hline & \multicolumn{2}{|c|}{$\begin{array}{l}t=3.25[\mathrm{DF}=58], \mathrm{p}=0.002 \\
\text { Significant change }\end{array}$} & \multicolumn{2}{|c|}{$\begin{array}{l}\mathrm{t}=0.20 \text { [DF 64], } \mathrm{p}=0.84 \\
\text { Non significant change }\end{array}$} \\
\hline \multirow[b]{2}{*}{ HDL cholesterol } & $51.53 \pm 11.25$ & $35.86 \pm 6.52$ & $50.3 \pm 6.96$ & $52.12 \pm 5.63$ \\
\hline & \multicolumn{2}{|c|}{$\begin{array}{l}\mathrm{t}=6.60[\mathrm{DF}=9.58], \mathrm{p}=0.004 \\
\text { significant change }\end{array}$} & \multicolumn{2}{|c|}{$\begin{array}{l}\mathrm{t}=1.17[\mathrm{DF}=64], \mathrm{p}=0.2 \\
\text { Non significant change }\end{array}$} \\
\hline \multirow[b]{2}{*}{ LDL cholesterol } & $88.03 \pm 21.03$ & $88.5 \pm 21.18$ & $91.69 \pm 11.42$ & $88.39 \pm 12.79$ \\
\hline & \multicolumn{2}{|c|}{$\begin{array}{l}\mathrm{t}=0.09[\mathrm{DF}=58], \mathrm{p}=0.9 \\
\text { Non significant change }\end{array}$} & \multicolumn{2}{|c|}{$\begin{array}{l}\mathrm{t}=1.11[\mathrm{DF}=64], \mathrm{p}=0.273 \\
\text { Non significant change }\end{array}$} \\
\hline \multirow[b]{2}{*}{ Triglycerides } & $148.73 \pm 24.54$ & $115.71 \pm 21.62$ & $147.12 \pm 21.53$ & $146.29 \pm 18.84$ \\
\hline & \multicolumn{2}{|l|}{$\begin{array}{l}\mathrm{p}=<.001 \\
\text { Significant chan }\end{array}$} & \multicolumn{2}{|c|}{$\begin{array}{l}\mathrm{t}=0.17[\mathrm{DF}=64], \mathrm{p}=0.8 \\
\text { Non significant change }\end{array}$} \\
\hline
\end{tabular}

Overall improvement in vasomotor symptoms was significantly higher in tibolone group in all follow up visits and after the completion of treatment. Also complete relief of vasomotor symptoms was significantly higher number in tibolone group compared to isoflavones group. Tibolone was a better drug to relieve the genitourinary symptoms compared to isoflavones. But continued and sustained use of tibolone was required to achieve symptomatic relief. Effect of isoflavones was very mild in genitourinary symptoms and no women had complete relief of symptoms. Very few patients had symptomatic improvement in joint pain after 1month in tibolone group. But with continued and sustained use, a significant proportion of patient reported relief of symptoms. In isoflavones group, effect was very mild. Only 2 patient reported partial improvement in joint pain.

Blood pressure records did not show any changes with the use of any of the drug(s) over 12 month. 2 patients had vaginal spotting with in 1 month of initiation of therapy. No Women on isoflavones had lesser incidence of breakthrough bleeding. Only recurrence of bleeding was noticed in those 2 women and endometrial thickness also was $<5 \mathrm{~mm}$. On the other hand incidence of breakthrough bleeding was higher in tibolone group (50\% in first month, $21 \%$ in 6 month and 14.3\% in 12 month). But on continued use incidence became less and very few recurrence of bleeding was found. No patient had endometrial thickness $>5 \mathrm{~mm}$ with breakthrough bleeding in tibolone group.No significant difference was found in endometrial thickness after 12 month from the initial thickness in both groups. Women using tibolone showed significant decrease in triglyceride (22.44 $\pm 3.9 \%$ from baseline) and total cholesterol $(11.28 \pm 4.81 \%$ from the baseline) and decrease in HDL cholesterol $(30.67 \pm 5.25 \%)$. But no significant change was found in LDL cholesterol in tibolone group. In isoflavones group, no significant change in lipid profile was found.

\section{CONCLUSION}

In the present comparative study, efficacy of tibolone was found to be significantly better than the isoflavones in alleviation of postmenopausal symptoms. Tibolone can be considered as an effective alternative to conventional hormone replacement therapy. On the other hand iso flavones have modest effect on vasomotor symptoms and very minimal effect on other postmenopausal symptoms.

\section{REFERENCES}

[1] Hammer M, Christau S, Nathorst-Boos J, Rud T, Garre K, A double blinded, randomized trial comparing the effects of tibolone and continuous combined hormone replacement therapy in postmenopausal women with menopausal symptoms, Br J Obstet Gynecol 105:904,1998.

[2] AL-Azzawi F, Wahab M, Habiba M, Akkad A, Mason T, Continuous combined hormone replacement therapy compared with tibolone, Obstet Gynecol 93:258, 1999.

[3] Laan E, Van Lunsen RHW, Everaerd W, The effects of tibolone on vaginal blood flow, sexual desire and arousability in postmenopausal women, Climacteric 4:28, 2001.

[4] Botsis D, Kassanos D, Kalogirous D, Antonious G, Vitoratos N, Karakitsos P, Vaginal ultrasound of the endometrium in postmenopausal women with symptoms of postmenopausal urogenital atrophy on low dose estrogen or tibolone treatment: a comparison, Maturitus 26:57, 1997. 
[5] Castelo -Branco C, Vicente JJ, Figueras F, Sanjuan A, Martinez de Osaba MJ, Casals E, Pons F, Balasch J, Vanrell JA, Comparetive effects of estrogens and tibolone on bone, lipid pattern and sexuality in postmenopausal women, Maturitus 34:161,2000.

[6] Egarter C, Topcuolu AM, Vogl S, Sator M, Hormone replacement therapy with tibolone: effects of sexual functioning in postmenopausal women, Acta Obstet Gynecol Scand 81:649, 2002.

[7] Genazzani AR, Benedek Jaszmann L, Hart DM, Andolsek L, Kicovic PM, Tax L,Org OD14 and the endometrium, Maturitus 13:243, 1991.

[8] Ginsburg J, Prelevic G, Butler D, Okolo S, Clinical experience with tibolone (livial) over 8years, Maturitus 21:71, 1995.

[9] Geusens J, Dequeker J, Gielen J, and Schot LPC, Nonlinear increase in vertebral density induced by a synthetic steroid (Org OD14)in women with established osteoporosis, Maturitus 1991;13:155-162.

[10] Rymer J, Effects of 8years of treatment with tibolone $2.5 \mathrm{mg}$ daily on postmenopausal bone loss, Osteoporos Int $12: 478$, 2001.

[11] Trevoux R, Dieulangard P, Blum A, Efficacy and safety of org OD 14 in the treatment of climacteric complaints, Maturitus 5:89,1983. 